\title{
Duelos fELICES, TEORÍAs ÁGILES ${ }^{1}$
}

\author{
Happy mournings, agile theories \\ Gabriel Gatti* \\ * Universidad del País Vasco/Euskal Herriko Unibertsitatea \\ g.gatti@ehu.eus
}

Palabras clave
Duelo
Teoría sociológica
Imaginación sociológica
Magisterio

Keywords

Mourning

Sociological theory

Sociological imagination

Teaching

\section{Resumen}

A partir de la revisión de los límites y los usos posibles de una categoría periférica en la obra de Alfonso Párez-Agote, la del duelo, el artículo hace un "elogio aplicado" de una forma de "hacer teoría investigando" (teoría caliente, teoría de alcance intermedio). El elogio aplicado, por mandato de coherencia, solo puede ser tal si estira los usos de la categoría en Pérez-Agote, si los lleva más allá. Así, además de proponer desindividualizar o socializar el concepto de duelo, el texto lanza una hipótesis sencilla: que el duelo puede ocupar existencias colectivas completas en el espacio y en el tiempo y que esas existencias colectivas son sociales, pero no sociales como cualquier otra.

\section{Abstract}

Through the review of the limits and possible uses of a peripheral category of Alfonso PérezAgote's work, that of mourning, the text makes an "applied praise" of a way of "doing theory through researching" (hot theory, middle-range theory). The applied praise can, by coherence, only be so if itself stretches the Pérez-Agote's uses of the category, if it takes it beyond. It means that, besides proposing the des-individualization or socialization of the mourning category, the article offers a modest hypothesis: that mourning can occupy complete collective lives in space and time and that those collective lives are social, but not social as any other.

Gatti, G., 2015, "Duelos felices, teorías ágiles", en Papeles del CEIC, vol. 2015/3, no 135, CEIC (Centro de Estudios sobre la Identidad Colectiva), Universidad del País Vasco, http://dx.doi.org/10.1387/pceic.15185

\footnotetext{
${ }^{1}$ Algunos de los argumentos utilizados en este trabajo proceden de las reflexiones emprendidas con el equipo del proyecto "Mundo(s) de víctimas" (CSO 2011-22451). Con la misma intención -el reconocimiento- debo citar otras tres instancias que posibilitaron trabajar en esto y hacerlo cómodo: en el largo plazo, el grupo consolidado del Sistema Universitario Vasco (IT706-13), en el corto el LALS de la Universidad de California en Santa Cruz, donde redacté la versión final de este texto, en el cortito pero imprescindible para arrancar, las Jornadas "A propósito de la obra de Alfonso Pérez-Agote", celebradas en noviembre de 2014 en la Universidad Complutense de Madrid, donde presenté un primer esbozo de este texto.
} 


\section{Homenajes Debidos. Nota introductoria (BREVE,} DESCUIDEN)

Un artículo, por corto que sea, dentro de un número de revista o un libro que homenajea a alguien debe dejar claro en algún punto por qué y cómo encaja con algo característico del homenajeado o de su obra. O de ambas. No es raro que la conexión se fuerce: porque cómo él o ella toco los mismos temas, porque cómo él o ella pisé los mismos lugares, las mismas escuelas, padecí las mismas épocas. No es caso en lo que me une a Alfonso Pérez-Agote: nacimos en partes del planeta casi opuestas, vivimos y sufrimos las cosas de algunas de los años más convulsos del siglo XX habitando lugares muy distintos, estudiamos en distintas instituciones, lo hicimos en distintas épocas. Pero coincidimos en otras épocas y lugares que sin ambages me obligan a decir que tiene conmigo una relación de verdadero magisterio, de los que valen la pena, esos que no se notan hasta que uno no tiene más remedio que asumir que han dejado señales muy marcadas. Fue transmitiéndose de manera afable, divertida, conviviendo con él en las largas sesiones de investigación del grupo que compartimos durante años, en una época con cosas que tenían que ver con ese estado de neurastenia que llaman tesis doctoral, en otra investigando otras cosas (jóvenes, vascos, desplazados, lenguas, precarios, migrantes, víctimas, violencia...).

En cualquiera de esos ejercicios incorporé, mal o bien, pero incorporé, una manera de mirar, de hacer teoría (García Selgas, 2015), la que ha sido su sello en la Academia, la que puede decir que constituye escuela. Debe serlo: se le sigue transmitiendo hoy a gente que ni le conoce. El propio Alfonso Pérez-Agote ha hablado de su apuesta en unos términos, los térmicos, esos que diferencian entre teorías calientes o frías en función de la intensidad de la integración entre la mirada teórica y el trabajo empírico, una actualización de las viejas teorías de alcance intermedio (Merton, 1984), que aunque pueda ser que sea criticable (cf. en este mismo volumen de Papeles del CEIC la contribución de Fernando García Selgas (2015)), identifica maneras de hacer regidas por ecuaciones que exigen a quien las aplica mezclar trabajo teórico y empírico y aderezar la ensalada con intensidad analítica y diría que con algo -para mí mucho- de "incorporación al objeto": hacer de la historia que uno cuenta historia propia, ver hasta qué punto y cómo lo que uno mira es propio para poder contarlo mejor. Es posible que con ese "algo" Alfonso discrepe. Sin embargo, no puedo si no ver eso en todo lo que ha 
trabajado: intensidad e incorporación al objeto; hace las historias suyas, las filtra por su cuerpo para entenderlas: así con vascos en Euskadi, con jóvenes sin emancipar, con la secularización, con la idea de nación o la de dios, o Dios, con los vascos en Argentina... Otra cosa, y eso más que discrepancia es cuestión de obligaciones de época, es que el relato de estas incorporaciones deba quedar - para él- en las bambalinas de la investigación social y para mí lo constituye y debe informarlo, desde la primera a la última coma. Sobre este asunto, que no es solo de epistemologías, regreso en el final de este pequeño texto.

Lo que sigue es un canto al magisterio, a una cierta forma à la francesa de entender la sociología (duda metódica, constante duda metódica, cartesianismo y pesadez analítica, sin freno) y a una cierta forma de interpretar el trabajo teórico en sociología. De lo que se trata de hacer la enunciación es fácil: la teoría es una herramienta que solo sirve si es ágil. Requiere de conceptos de viene y va, cambiantes según lugares y contextos, transversal a muchos objetos y situaciones $-y$ por eso en algo universal - pero también adecuada para la singularidad de cada situación $-y$ por eso profundamente local-; como glosarios pero en constante, imparable, reelaboración y crítica. Trabajaré aquí con un concepto, el duelo. Mostraré primero algunos significados originales, daré luego muestras de cómo el trabajo de Alfonso Pérez-Agote los reelabora y aplica. Y seguiré la rueda para que gire y gire: reelaborando lo que hizo Alfonso para aplicarlo a otros lugares y problemas.

\section{EL DUELO COMO CATEGORÍA DE ANÁLISIS SOCIOLÓGICO}

La referencia al concepto de duelo en Sigmund Freud aparece brevemente en tres trabajos de Pérez-Agote, uno sobre la comunidad de descendientes de exiliados vascos asentada en el Río Carabelas, en las estribaciones del Río Tigre, allá lejos, en Argentina (Pérez-Agote et al., 1997), otro sobre los grupos de autoayuda organizados por mujeres madres de personas muertas en accidentes de tráfico (Pérez-Agote, 2004) y finalmente un tercero sobre los ritos funerarios en el nacionalismo vasco radical (Pérez-Agote, 2008). En los tres, el acercamiento al concepto de Freud es parecido y también lo es la ampliación del radio de alcance de la categoría que propone aquel. Aunque en cada caso se aplica a situaciones en apariencia muy distintas la reelaboración que desarrolla apuesta por lo mismo: sacar al concepto de la encerrona del individuo solitario y socializarlo. Me apoyo ahora en 
el trabajo de 2008, que es donde esta reelaboración aparece -a mi conocimiento al menos- más desarrollada.

Para Freud, resume Pérez-Agote, el duelo es (1) un estado psíquico individual, que aqueja al sujeto afectado por una pérdida; (2) un estado psíquico provisorio, que ocupa a ese sujeto en absoluto mientras digiere la pérdida, la ausencia, de eso que desapareció, del afecto desaparecido. Es, en fin y en síntesis, un modo de administrar y de elaborar una ausencia que se si se hace bien, dura un tiempo, el que requiere no tanto regresar al estado previo a la muerte o al dolor $o$ al sufrimiento bruto que provocó el duelo, pero sí a uno donde sea posible hacer un nuevo equilibrio, regresar a un mundo gobernado de nuevo por el principio de realidad. Entonces el individuo ya no estará arrasado por la pérdida: la aceptará pues habrá reemplazado al objeto amado por otro. Restituyó la normalidad, escapó del agujero. No le queda otra: en él, piensa Freud, nadie puede vivir demasiado tiempo sin reventar. Si lo hiciese, si la pérdida no fuese aceptada, si no se abriese el camino a construir una nueva realidad, si el sujeto, en fin, se instalase en la ausencia y la quisiese habitar, el duelo se volvería patológico. La vida - de ese modo una pura anomia-sería imposible, carecería de sentido y de orden.

Nos encontramos entonces con una categoría dura, con un enorme potencial pero que presenta, en la interpretación y uso de Freud, tres fuertes limitaciones. En cuanto al potencial, pasa por su capacidad de dar cuenta de un estado del ser fuera del "estado debido del ser". A la par de otros conceptos viejos, como el siempre infrautilizado en sociología de anomia o el lamentablemente en estado larvario para esta disciplina de liminalidad, el duelo recorta un tiempo extraño, ajeno a la rutina pero sin embargo con vida. Las restricciones por su parte, son tres, y son fuertes. Una hace a su encierro -en Freud- entre las paredes de la existencia individual; la otra, a que se refiere a un estado necesariamente provisorio -a riesgo en caso contrario de devenir "patológico"- . De esas dos me ocupo en breve, de la tercera no, con lo que apenas la menciono: lo despiadado de las herramientas teóricas de cuando las ciencias sociales nacieron con lo sinsentidoso (Gatti, 2005).

Tras identificar el potencial, Alfonso Pérez-Agote sociologiza la categoría, primero historizándola, es decir, cuestionando la universalidad de la institución social del duelo ("el duelo como mecanismo que el entorno social utiliza para permitir que una persona recupere la primacía de su 
principio de realidad varía en el espacio y con el tiempo, como nos lo muestran los antropólogos sociales" (2008: 429)), luego colectivizándola:

“La definición de Freud se refiere (...) a un estado psíquico personal ocasionado por la pérdida de un objeto amado. Pero, como podemos observar a lo largo de nuestras vidas, el duelo tiene también una dimensión social que alcanza la escala familiar, la del vecindario en el mundo rural tradicional, e, incluso, esferas sociales más amplias" (2008: 427).

Lo primero es necesario, y en algún aspecto, que no desarrollaré aquí, ejemplo a seguir ${ }^{2}$. Lo segundo constituye el hallazgo fuerte del trabajo de Pérez-Agote en la materia: una categoría antes ceñida al limitado territorio del individuo adquiere valor sociológico al construirla como herramienta de análisis empírico para entender el trabajo de una comunidad de actores sociales cuando afronta situaciones de perdida compartidas. El trabajo de reelaboración que propone pasa, primero, por sacar el concepto del contexto original (un individuo que sufre una pérdida) ${ }^{3}$ e identificar después situaciones donde ese proceso (sufrir una pérdida) constituye sociedad. Así el exilio, cuando, en el agujero que deja la pérdida, por distancia y expulsión de la tierra originaria, se hace un colectivo; así también los rituales de muerte de una comunidad política, en este caso, la nacionalista vasca, cuando la ausencia del héroe, sacrificado, dispara la organización de una comunidad en el hueco que aquel hace visible; así finalmente, los "grupos circunstanciales" (Vilain y Lemieux, 1998) integrados por personas afectadas por accidentes de tráfico, organizados con solo un dato en común, la pérdida trágica pero circunstancial de un ser querido. De este modo, a una escala mayor que la del solo individuo, la escala del pequeño grupo, de la comunidad de cercanos, se organiza un trabajo de gestión de la pérdida compartida por el que se constituyen colectivos que no existían ni antes de la catástrofe que originó la pérdida, ni sobre todo antes del trabajo que se lleva adelante para su gestión.

\footnotetext{
${ }^{2}$ En un contexto en el que parece imparable la transnacionalización de ciertas figuras para dar nombre y pensar el dolor, personajes sociales como el de detenido-desaparecido o el de familiar de detenido-desaparecido, son ahora pensados como categorías universales, tanto como lo son algunos de los sufrimientos que se les asocian, caso del duelo (en este caso, "duelo no resuelto" o "duelo inacabado" (HRW, 2013; Al, 2014)), que se presume indeleble a todo afectado por estos dolores, aquí o allá, entonces o ahora.

${ }^{3}$ Lo que han hecho otros muchos, aunque no a mi conocer desde el campo de la sociología. Personalmente, he trabajado con los textos de Rousseaux y Olmo (2005) o Gómez Mango (2006) desde el psicoanálisis.
} 
Ampliación, pues, del campo de batalla del duelo, del alcance de la categoría. Pero a mi juicio puede irse más allá, subir de escala: el duelo puede proponerse como una categoría para definir y describir situaciones "estructuradas en la desestructura", esto es, mucho más que una mera excepción en el tiempo y en el espacio o si se quiere, una excepción convertida en norma, una "excepcionalidad social compartida" (PérezAgote, 2008: 428) pero que dura. Voy a hacer entonces sobre el de PérezAgote el trabajo de estiramiento que él mismo hace sobre la categoría en Freud. Propongo sobre todo elevar la escala, el alcance de la categoría, tanto en el espacio como en el tiempo: es una categoría totalizadora en ciertas situaciones. Me interesa entonces preguntarme por cómo pensar teóricamente las situaciones en las que los duelos se convierten en nichos que se habitan de manera permanente. Es esa mi hipótesis. En realidad, al hacerlo, al estirar las categorías, moldearlas, quebrarlas para poder usarlas, no sigo sino el mandato del trabajo de quien ahora quiero criticar.

\section{El DUELO COMO ESPACIO HABITABLE}

La primera prolongación de la categoría se mueve en el tiempo. Dice Alfonso Pérez-Agote que las situaciones de duelo están destinadas a ser superadas ¿Es necesariamente así? En el duelo, esta es la situación: un acontecimiento quiebra lo existente. Lo desajusta. La primera opción es pensar ese acontecimiento como un trauma, esto es, como una desestabilización que es profunda pero dura poco, y que hay instituciones con capacidad de regular los desajustes, competentes entonces para que a la desestabilización le siga la institucionalización de un nuevo equilibrio del sentido y de los hechos. Que hagan que pasado el tiempo todo regrese a su lugar; que se asimile, se normalice. La muerte de un cercano es un trauma; el duelo à la Freud, cuando se cierra y se resuelve, es una institución que permite gestionarlo. Aunque duela, lo torcido se endereza.

Pero hay otra opción: que al acontecimiento que quiebra -la catástrofe- no le suceda un nuevo orden y que la vida se instale en ese lugar. Es, dijo Ignacio Lewkowicz, "una dinámica que produce desmantelamiento sin armar otra lógica equivalente" (Lewkowicz et al., 2002: 154). O sea: lo que rompe, dura. Por siempre, sin reemplazo. Y la vida se instala allí, aunque parezca imposible: la anomia hecha norma, la ambivalencia hecha valor, la excepción permanente. Un duelo perpetuo. 
"Esta vez -dice de nuevo Lewkowicz- la inundación llega para quedarse" (ibídem).

La segunda prolongación de la categoría se despliega en el espacio. Me interesaría en ese sentido pensar en situaciones en las que en torno a este impasse, el duelo, se construye sentido y se hace de manera estructurada, aunque a priori no lo pueda ser. En Vidas precarias. El poder del duelo y la violencia (2006), Judith Butler se propone analizar la construcción de vida política en situaciones que la imposibilitan, generalmente las de máxima exposición a la violencia, cuando estamos expuestos a, dice, la vulnerabilidad de la pérdida "y al trabajo del duelo que le sigue" (ibídem: 45). ¿Es posible en ellas construir una comunidad? A estas situaciones ¿se les puede dar sentido sin salir de ellas? ¿Cómo "reimaginar la posibilidad de una comunidad sobre la base de la vulnerabilidad y la pérdida" (ibídem: 45)? La pérdida nos suele reunir en tenues nosotros, pensados siempre como parciales y provisionales ("cuando perdemos a ciertas personas o cuando hemos sido despojados de un lugar o de una comunidad podemos simplemente sentir que estamos pasando por algo temporario (sic), que el duelo va a terminar y que vamos a recuperar cierto equilibrio previo" (ibídem: 48)). Así es, creemos que tras ese hueco volverá el colmo, el lleno, el orden. Sin embargo, el duelo puede ser eterno y puede ser también colectivo, armar vínculo y no tenue ("El duelo - sigue Butler- permite elaborar en forma compleja el ser nítido de una comunidad política" (ibídem)). El árido suelo de la teoría heredada no permite esta opción: la ausencia no constituye, lo sin (Rancière, 1995), es solo falta, pérdida. Precariedad. Solo cabe salir de ahí porque ahí no se puede estar. Nuda vida (Agamben, 1998) y nada más.

O no: la vida de los que están en pena puede seguir siendo en ese estado, o por eso apuesta Butler. Las "zonas 'invivibles', 'inhabitables' de la vida social, sin embargo, están densamente pobladas por quienes no gozan de la jerarquía de los sujetos" (2002: 19-20), dice. Sea, de nuevo, una situación, un momento, de duelo, cuando uno "está entregado a algo que no controla y se descubre fuera de sí, en desacuerdo consigo mismo" (Gómez Mango, 2006: 54), desposeído de uno mismo ¿Hay vida en la desposesión? Pensemos en situaciones sociales que puedan ser descritas con ese término, desposesión: los sin derechos a tener derechos, la vida social en la basura, los refugiados en un campo, los afectados por una catástrofe, los perdidos en el desierto o en el mar 
buscando alcanzar otro país... Montones de sujetos frágiles respecto a ciertos marcos normativos específicos (el del cuerpo, el de la identidad, el de la vida íntegra, el de lo nacional, el de los papeles de identidad...) frente a los que se define su situación como de pérdida y su identidad como la de un desolado, un despojado. $O$ un excluido, por qué no.

La dignidad heredada, ilustrada y socializante, nos invita y empuja a pensar que ante todo eso lo deseable es hacer que estos sujetos (re)ingresen a nuestra idea de lo íntegro. Pero hay quizás un error de interpretación en esa lectura: estos sujetos están fuera de lo que define la vida y si se los saca de ese lugar en nombre de que alli no hay vida, de que en la miseria, la muerte, el campo de concentración, el Mediterráneo cuando se lo cruza, el campo de refugiados, cualquier espacio de excepción... no se puede existir, no solo se los convierte en otra cosa, sino que se aplica un imperial y reductor concepto de vida, de norma, de orden. Realmente, ¿no hay nada de vida en los reinos de la anomia? ¿Nada de vida colectiva? ¿No tienen vida estos espectros, aunque sea otra forma de vida?

He trabajado sobre las comunidades de sentido que se constituyen en Argentina en torno a la figura del detenido-desaparecido, una figura no dueleable, pues ni muerto ni vivo, pues ni ausente ni presente (Gatti, 2008) y que sin embargo articula a su alrededor verdaderos mundos de vida. En equipo ${ }^{4}$, hemos detectado que en torno al personaje social de la víctima se ordenan en España existencias completas y tramas de sentido complejas, muy abarcativas, totalizantes, plenas de dolientes y de duelentes, pero también de expertos, de oficios, de técnicas, de protocolos. Cerca de ese quipo, la tesis doctoral de María Martínez (2015) permite intuir formas de agencia entre colectivos de mujeres posicionados en lugares de vulnerabilidad radical, lugares a priori de no existencia y sin agencia pero henchidos de ambas, o el trabajo de fin de master de Ander Mendiguren (2015), que en las rutinas de supervivencia de un grupo de enfermos de SIDA en Bilbao perfila, en la estela de trabajos como los de Joao Biehl (2005) o Philippe Bourgois (2009), la rara vida de las zonas de abandono, la de los excluidos zombi. Hay más: algo antes y más lejos, Michel Agier (2008) ha sabido ver cómo en los campos de refugiados - lugares de anomia - se estructuran rutinas - normasy agencia en sujetos que no la tienen. En esos espacios de excepción,

\footnotetext{
${ }^{4}$ Junto a los miembros de "Mundo(s) de víctimas", proyecto ya citado. 
sujetos sin (no ciudadanos), muestran no solo lo que suelen mostrar ("Ios límites de la vida y los límites de la política" (ibídem: 45)) sino también en sus "acciones, tácticas de resistencia y tomas de palabra (...) [manifiestan] un nuevo tipo de lucha política sin ciudadanía previamente reconocido" (ibídem). La tesis, en fin, no es distinta a la de Veena Das cuando trabaja sobre las comunidades de dolor (2008) o a la de Alexander Weheliye cuando lo hace sobre las posibilidades de vida en la nuda vida (2014): Ios sin agencia -esclavos, no humanos, mujeres violadas, desaparecidos, zombis...- tienen agencia, pero es cierto que es otra bien distinta a lo que la teoría heredada determina (Gatti, en prensa).

¿Puede el duelo servir de herramienta para comprender todo eso? Sí, sin duda. Un buen ejemplo es el trabajo de Cecilia Sosa (2014), que radicaliza la hipótesis que estoy proponiendo seguir: en Argentina, analiza Sosa con mucha sutileza y algo de perversión, entre los afectados por la desaparición forzada de personas en la dictadura 19761983, una primera generación compuso una históricamente muy novedosa narrativa, la del duelo compartido. Conformó en torno a ella una "comunidad biológica de víctimas": era, añade, una "asamblea de ancestros de víctimas" que terminó por instaurar en Argentina una "fundación biológica de las demandas de justicia": para poder reclamar reconocimiento y reparación y hacerlo con legitimidad solo cabía hablar desde el dolor del duelo que afecta a los unidos por sangre a los sufrientes originarios, los desaparecidos. Pero la narrativa se instituye y deviene política pública. y suceden dos perversiones, dos queerificaciones en la propuesta de Sosa: una, que las jóvenes víctimas (la "segunda generación de sobrevivientes" (ibídem: 5)) "iluminan una nueva idea de comunidad basada en los placeres del duelo compartido" (ibídem: 74). Y dos, y sobre todo, que el duelo se socializa. La comunidad ya no es la biológica de víctimas, sino la "no biológica del duelo" (ibídem: 73): la vieja narrativa sale del pequeño grupo de afectados directos y deviene nacional; el duelo por los militantes o héroes o mártires o víctimas desaparecidas en dictadura no es solo materia privada, es constitutivo de una forma de entender la nación y la argentinidad común hoy a la ciudadanía en su conjunto. El duelo, en fin, constituye lazo social, no solo ya comunidades de corto alcance; va más allá, se extiende a la sociedad toda, supera los límites del círculo de quienes sufren la pérdida, abarca incluso a aquellos que no están ligados por 
lazos de parentesco con los ausentes, desaparecidos o muertos. El principio de realidad gana de nuevo, pero en forma de lo que en Freud lo negaba.

En conclusión (o casi): en la forma, he partido de un acuerdo con el trabajo de Pérez-Agote para llegar al final a un desacuerdo. El acuerdo es que las situaciones de duelo son situaciones sociales, que hay que elevar la escala de trabajo de Sigmund Freud; la diferencia es que no puedan durar y que sean sociales "como las otras": la burbuja de excepcionalidad en que consiste el duelo puede estabilizarse y en torno a eso, aun siendo patológico, se estructuran mundos de vida con sentido, aunque es verdad, con un sentido otro de lo que pensábamos que era lo "con sentido". En conclusión (ya sí): en el fondo, las herramientas de la teoría están para usarse y romperse. Creí ver en eso una enseñanza que he aplicado.

\section{Homenajes Queridos. nOtA de CIERRE (TAMBiÉn BREVE, DESCUIDEN)}

Cierro este texto recordando dos momentos, ya lejanos. Los dos tienen que ver con el magisterio invisible e intenso de Alfonso. Ambos dan cuenta de aprendizajes importantes, vitales y académicos, no soy capaz de distinguir mucho; en ambos, aparecen duelos, creo que resueltos. O si no, de los que se vive en con cierto placer. Ya les digo: seré breve, no tengan miedo.

El primero es de 1996, en Evora, Portugal, el primer congreso al que asistí de la Association Internationale des Sociologues de Langue Française, mi primer congreso internacional, para mí un baño en muchas iniciaciones. Fui a la AISLF y a Evora de la mano, no literalmente, sépase, de Alfonso. Hablamos allí de varias cosas de las que en la rutina de un departamento y de una relación director de tesis-doctorando no se hablan, entre otras, de historias de infancia, de clases sociales, de educaciones sentimentales, de personajes centrales para uno y otro en unas y otras. Supe entonces de la condición de hijo menor de Alfonso, también de su condición de hijo póstumo, del padre que no estuvo porque no pudo estar, y de la contundencia de los afectos de su madre. Supe también de cómo gestionó su muerte, del duelo, y de cómo lo incorporó luego a su trabajo sobre el País Vasco y la violencia política, de cómo en ese proceso, pese al mucho dolor, supo divertirse, esto es, cómo 
le sacó partido porque distrajo al monstruo mirándolo desde otro lado. Hasta mucho después, esas conversaciones flotaban en mi memoria como buenos momentos, buenas charlas, nada directamente académico. Vida nomás.

El segundo son en realidad muchos momentos, entre 2005, más o menos, hasta 2008 o 2010. Coincide con mis elecciones de trabajo postdoctorales, lejos de los problemas de la identidad en el País Vasco, y cada vez más cerca de América Latina y, dentro de los asuntos de ésta, de mi conversión (matter of destiny?) en desaparecidólogo viajero. Arrancaban por entonces las peleas - que siguen: es una categoria dura, pero me divierto mucho con ella - con la categoría de desaparecido. Lo hice primero para darle forma a un texto elaborado para las reuniones del grupo de teoría sociológica, otra iniciación que le debo a Alfonso (Gatti, 2005), texto que junto a muchas más cosas y personas y decisiones luego se tradujo en un primer libro sobre este monstruoso $y$, ¡ay!, divertido tema (Gatti, 2008). De él, solo un dato: trabajo desde la distancia analítica que marca mi estómago con un tema que, sin embargo, me constituye: la desaparición forzada en general como asunto sociológico, la desaparición forzada concreta de mi padre y de mi hermana como mi asunto. Bien o mal, hice un trabajo de análisis que no pasó por el prefijo psico sino por el socio. Charlando alguna vez de eso con Alfonso, me dijo: "Tú ya has hecho el duelo, y lo has hecho con esto, sociología". Duelos felices, divertidos. Teorías ágiles. Sociología nomás.

\section{Bibliografía}

Agamben, G., 1998, Homo sacer. El poder soberano y la nuda vida, PreTextos, Valencia.

Agier, M., 2008, Gérer les indésirables. Des camps des réfugiés au gouvernement humanitaire, París, Flammarion.

Amnistía Internacional, 2015, "Disappearances". https://www.amnesty.org/en/what-we-do/disappearances/. Última consulta: 15 de octubre de 2015.

Biehl, J., 2005, VITA: Life in a Zone of Social Abandonment, Berkeley, University of California Press.

Bourgois, Ph., Schonberg, J., 2009, Righteous dopefiend, Berkeley, University of California Press.

Butler, J., 2002, Cuerpos que importan, Buenos Aires, Paidós. 
Butler, J., 2006, Vidas precarias. El poder del duelo y la violencia, Barcelona, Paidós.

Das, V., 2008, Sujetos del dolor, agentes de dignidad, Bogotá, Universidad Nacional de Colombia-Pontificia Universidad Javeriana.

García Selgas, F., 2015, “El calor de la teoría”, en Papeles del CEIC. International Journal on Social Identity Research, 2015/3 (número especial dedicado a Alfonso Pérez-Agote), $n^{\circ}$ 134, CEIC (Centro de Estudios sobre la Identidad Colectiva), Universidad del País Vasco, http://dx.doi.org/10.1387/pceic.13294.

Gatti, G., 2005, "La teoría sociológica visita el vacío social (o de las tensas relaciones entre la sociología y un objeto que le rehúye)", en A. Ariño (coord..), Las encrucijadas de la diversidad cultural, Madrid, CIS, pp. 177-202.

Gatti, G., 2008, El detenido-desaparecido. Narrativas posibles para una catástrofe de la identidad, Montevideo, Trilce.

Gatti, G., en prensa, "La hipótesis V. Las víctimas y su victoria sobre el poderoso (y muy agencial) ciudadano", en B. Tejerina y G. Gatti, Pensar la agencia en crisis.

Gómez Mango, E., 2006, "La desaparición, el duelo, la cultura”, en La desolación. De la barbarie en la sociedad contemporánea, Montevideo, Ediciones de la Banda Oriental.

Human Rights Watch, 2013, "Mexico: crisis of enforced Disappearances". Documento en red disponible en https://www.hrw.org/news/2013/02/20/mexico-crisis-enforceddisappearances. Última consulta: 15 de octubre de 2015.

Lewkowicz, I., Cantarelli, M. y Grupo Doce, 2002, Del fragmento a la situación. Notas sobre la subjetividad contemporánea, Buenos Aires, Altamira.

Martínez, M., 2015, "Identidades feministas en proceso", tesis doctoral inédita, Universidad del País Vasco/Euskal Herriko Unibertsitatea.

Mendiguren, A., 2015, "Aproximación a la exclusión zombi", TFM inédito, master Modelos y áreas de investigación en ciencias sociales, Universidad del País Vasco/Euskal Herriko Unibertsitatea.

Merton, R. K., 1984, Teoría y estructura sociales, México D.F., FCE.

Pérez-Agote, A., 2004, "Self-fulfilling prophecy and unresolved mourning: basque political violence in the twenty-first century", en B. Aretxaga (coord.), Empire and Terror: nationalism-postnationalism in the new 
millennium, Center for Basque Studies, University of Nevada, Reno, pp. 177-198.

Pérez-Agote, A., 2008, "La profecía y los rituales de duelo en el nacionalismo vasco radical", en I. Mendiola (coord.), Textos y pretextos para repensar lo social: libro homenaje a Jesús Arpal, Servicio Editorial Universidad del País Vasco, Leioa, pp. 413-434.

Pérez-Agote, A., Gurrutxaga, A., Azkona, J., 1997, Mantener la identidad: los vascos del río Carabelas, Servicio Editorial Universidad del País Vasco, Leioa.

Rancière, J., 1995, La mésentente. Politique et Philosophie, Galilée, Paris.

Rousseaux, F., Olmo, D., 2005, "El duelo, lo inconsciente, lo colectivo", documento inédito.

Sosa, C., 2014, Queering acts of mourning in the aftermath of Argentina's dictatorship. The performances of Blood, Tamesis, Londres.

Vilain, J.-P., Lemieux, C., 1998, "La mobilisation des victimes d'accidents collectifs. Vers la notion de 'groupe circonstanciel'", en Politix. 11/44, pp. 135-160.

Weheliye, A., 2014, Habeas Viscus. Racializing Assemblages, Biopolitics and Black feminist theory of the Human, Duke UP, Durham. 